\title{
APLICACIÓN DE NUEVAS TECNOLOGÍAS AL ESTUDIO DE POSICIONES DE LA GUERRA CIVIL ESPAÑOLA EN PINTO (MADRID)
}

\author{
Ángela Crespo Fraguas y Miguel Ángel Díaz Moreno ${ }^{1}$ \\ Mercedes Farjas Abadía, Guillermo Martínez-Pardo Gil, \\ Esther Alfonso Carbajosa y Carlos Ruíz Serrano²
}

\section{Resumen:}

Los trabajos de prospección Ilevados a cabo en el término municipal de Pinto (Madrid) han permitido localizar diferentes enclaves con restos arqueológicos relacionados con las operaciones militares realizadas en torno a la capital durante la Guerra Civil Española (1936-1939). Para su identificación y documentación se recurrió al uso de GPS durante las batidas de prospección así como a fotografías aéreas de diferentes periodos que ayudaron en el proceso. En colaboración con investigadores de la Universidad Politécnica de Madrid se aplicaron en una de las zonas de mayor relevancia, el yacimiento de "Los Yesares", diversas técnicas con el objetivo de conseguir una representación espacial lo más completa posible. Estas técnicas incluyeron levantamientos topográficos para realizar cartografias a diferentes escalas, la captación de imágenes mediante vuelos UAV (Unmanned Aerial Vehicle) y el uso de escáneres terrestres para la obtención de representaciones 3D.

\section{Palabras Clave:}

Guerra Civil, Topografía, Escáner laser, Fotogrametría, Unmanned Aerial Vehicle.

\section{Abstract:}

The surveys carried out in the municipality of Pinto (Madrid) have enabled us to locate various structural remains linked to the military operations that took place around the capital during the Spanish Civil War (1936-1939). In order to identify and record them, surveys were complemented with the use of GPS and air photographs from different time periods. Afterwards, and in collaboration with researchers from various universities, further methods aimed at generating a complete special representation of the area were applied directly to one of the sites which produced the best results, known as "los Yesares". These methods include topographic mapping that resulted in cartographic material at different scales, the photographic recording with flying Unmanned Aerial Vehicles, and the use of land scanners and GPS-corrected photogrammetrics with which to obtain 3D models.

1 Equipo de Cota 667- Arqueología y Patrimonio - pintocota667@gmail.com

2 Universidad Politécnica de Madrid - m.farjas@upm.es 


\section{Key words:}

Civil war, Topography, laser scanner, photogrammetry, Unmanned Aerial Vehicle.

\section{Introducción}

Al igual que la investigación de otros periodos, la Arqueología de la Guerra Civil es, en esencia, multidisciplinar. En ella la intervención de especialistas de distintos ámbitos no puede sino enriquecer nuestras perspectivas sobre el fenómeno histórico. En este sentido, la utilización de nuevas tecnologías aporta, en el ámbito metodológico, posibilidades de aplicación sobre los restos que abarcan aspectos desde la interpretación de estructuras inmuebles hasta la reconstrucción y comprensión de los paisajes del conflicto, mientras que en el aspecto de la gestión de la memoria del mismo ayudan en un campo tan relevante como es el de la divulgación. En el marco del “Proyecto de estudio y revalorización de los restos de la Guerra Civil Española (1936-1939) en el término municipal de Pinto, Madrid" se desarrollaron durante los primeros meses del año 2014 diferentes labores encaminadas a la localización y documentación de los vestigios del conflicto en dicha localidad. En una de las posiciones, Los Yesares, además de la prospección arqueológica, se han aplicado una serie de técnicas encaminadas a la modelización del territorio a diferentes escalas, que incluyen levantamientos cartográficos a escala 1:500 mediante el uso de topografía tradicional con tecnología GPS y vuelos UAV (Unmanned Aerial Vehicle), y otras orientadas a la reconstrucción tridimensional de las estructuras mediante el uso de escáner laser terrestre.

\section{Establecimiento de redes arqueológicas y realización de cartografía georreferenciada}

La extensión del yacimiento de Los Yesares es de 10 ha, que junto con las inmediaciones y el otro lado del arroyo Culebro forman un conjunto arqueológico de unas 18 ha que requerían de apoyo cartográfico. Dada las necesidades métricas del proyecto y la extensión que ocupa, se decidió realizar la cartografía en una escala 1:500, lo que conllevara una precisión de $10 \mathrm{~cm}$.

El proyecto arqueológico pretendía dejar establecida en la zona de forma permanente, una red arqueológica-topográfica local, para disponer de puntos con coordenadas precisas, enlazados con la red nacional REGENTE (Red Geodésica Nacional por Técnicas Espaciales) en los trabajos que iban a ser realizados y los futuros. Para enlazar con la geodesia nacional se utilizó el vértice geodésico más cercano, en concreto el vértice denominado "Cantueñas". La red local que se implantó en el área arqueológica consta de 9 vértices más el vértice geodésico de enlace "Cantueñas". Estos vértices se observaron con el método estático 
rápido contando con 3 receptores GPS de forma simultánea en 7 sesiones. En el proceso de diseño, implantación y observación se tardó 5 días. Posteriormente en gabinete se calculó y ajustó la red.
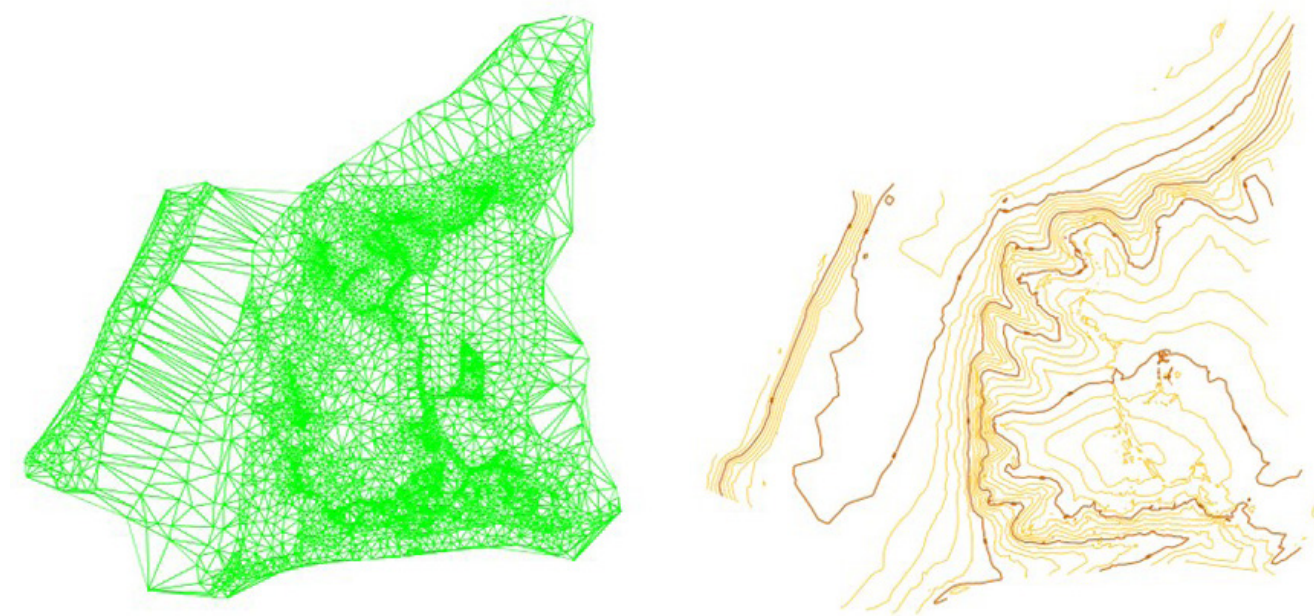

Fig.1. MDT y curvado de "Los Yesares".

El trabajo se realizó con técnicas espaciales GNSS (Global Navigation Satellite System) en concreto con la constelación NAVSTAR GPS. Para la toma de datos se utilizaron dos modelos de equipos GPS con similares prestaciones: el equipo GPS Leica 500 y el equipo GPS Leica 1200, aplicando dos metodologías diferentes con cada uno de ellos, de acuerdo a la fase del proyecto en el que se utilizaba. Los métodos de observación fueron los siguientes:

MÉTODO RELATIVO ESTÁTICO RÁPIDO: En esta metodología los equipos se montan sobre trípodes y se observa simultáneamente con al menos 2 receptores GPS obteniendo líneas base de distancias menores a 20 km, con precisión estándar de 5 a $10 \mathrm{~mm}+1$ ppm, y con tiempos de observación de 10 a 30 minutos. Este método necesita contar de antemano con un punto de coordenadas conocidas y un postproceso en gabinete.

MÉTODO RELATIVO DE FASE RTK (REAL TIME KINEMATIC): Se deja un receptor fijo como referencia en un punto conocido sobre el trípode y al menos otro móvil en mochila y bastón, se obtienen coordenadas en tiempo real con tiempos de observación de 3 a 6 segundos y una precisión de 20 mm +2 ppm.

El levantamiento arqueológico-topográfico, se realizó utilizando como referencia varios de los puntos de la red local y siguiendo el método RTK. La toma de datos se realizó con especial atención para llevar a cabo la documentación completa de los restos arqueológicos existentes, delimitando su contorno y su 
altitud/profundidad. Los detalles observados fueron tales como los 4 fortines, las trincheras, zanjas, pozos de tirador y los abrigos, también los límites naturales y artificiales, etc. Para todo ello se capturaron más de 8000 puntos, con precisión superior a. $4 \mathrm{~cm}$. Los puntos que no cumplían este requisito de precisión, se desecharon directamente en campo, y no fueron grabados en el proceso de observación.

Las coordenadas obtenidas por métodos GPS se capturaron en el sistema de referencia geodésico WGS84, no obstante para la representación cartográfica de este proyecto se requerían coordenadas en el Sistema de Referencia Geodésico (SRG) oficial en España, que es el ETRS89 (European Terrestrial Reference System 1989), y alturas ortométricas a partir del modelo geoidal EGM08-REDNAP (Earth Gravitational Model 2008 y el marco de referencia vertical dado por la Red Española de Nivelación de Alta Precisión); debiendo calcularse finalmente las coordenadas planimétricas de todos los puntos en la proyección UTM (Universal Transversa Mercator) huso 30.

Las coordenadas UTM huso 30 con alturas ortométricas de todos, se importaron al programa TopoCal para generar el MDT (Modelo Digital del Terreno) mediante una malla triangular. A partir del MDT se realizó el curvado del terreno con curvas de nivel cada metro y curvas maestras cada 5 metros.

Desde el programa de diseño gráfico AutoCad, tras importar el curvado, el MDT y los puntos con coordenadas UTM huso 30 y alturas ortométricas, se procedió a realizar la edición de la cartografía a escala 1:500 del yacimiento arqueológico de la Guerra Civil "Los Yesares" en Pinto, Madrid.

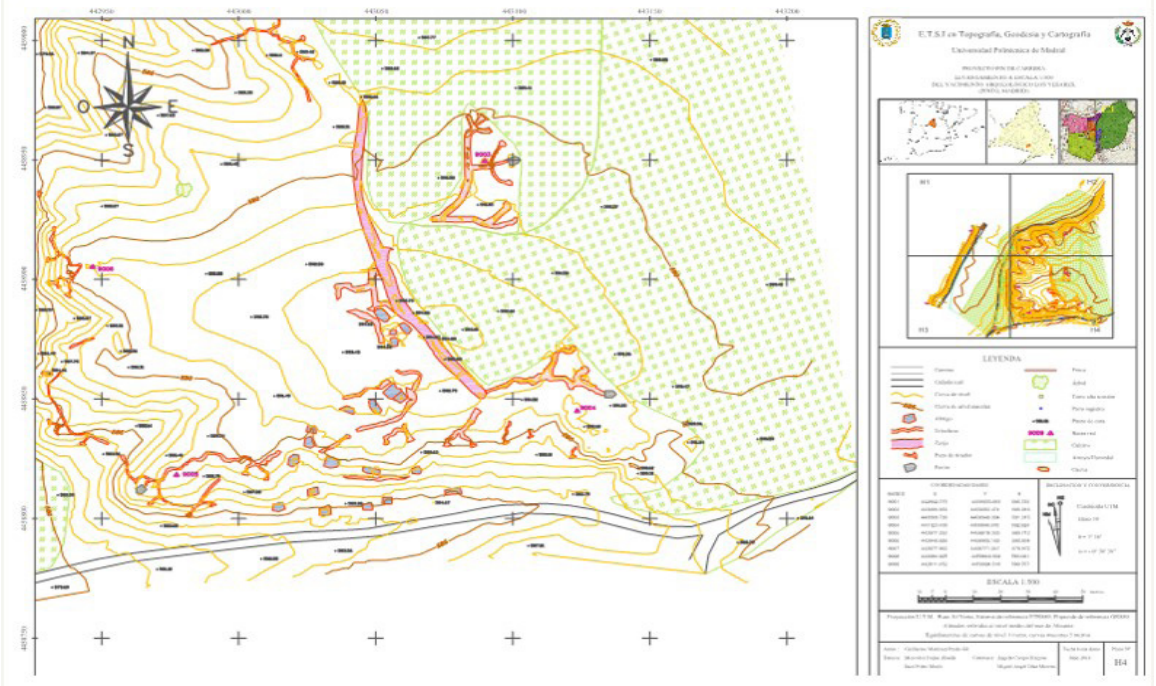

Fig.2. Edición cartográfica. 


\section{Metodología para la obtención de una ortoimagen mediante equipos UAV}

El proyecto que se describe a continuación consiste en la aplicación de las nuevas tecnologías de captura de imagen mediante drones y el tratamiento de las mismas mediante procesos fotogramétricos, en el ya mencionado yacimiento arqueológico de Los Yesares. Para obtener cartografía de una zona, tradicionalmente puede utilizarse la captura de fotografías aéreas de la misma, procesando la información mediante las tecnologías fotogramétricas.

La combinación de la fotogrametría con la aviación se realizó en la Primera Guerra Mundial, para llevar a cabo la interpretación de terreno enemigo por parte de ambos bandos. Utilizaban para ello dos cámaras que tomaban fotografías consecutivas con las que se realizaba una orientación relativa por medios exclusivamente ópticos y mecánicos. Con los sistemas de posicionamiento actuales y la evolución de la aviación y material fotográfico, hoy en día se realizan las orientaciones externas e internas del procesamiento fotogramétrico con mucha más precisión y facilidad.

Como se ha indicado la fotogrametría aérea requería la utilización de aviones, lo que conllevaba un gasto elevado y hacía que el método fuera solo rentable para grandes extensiones e importantes proyectos. En este campo se han incorporado nuevas tecnologías de captura aérea de imágenes, y en la actualidad se dispone de los denominados drones o UAV (Unmanned Aerial Vehicles, vehículos aéreos no tripulados), con los que es posible sobrevolar áreas de interés, a menor altura y sin necesidad de realizar una gran inversión económica, en comparación con la fotogrametría clásica a gran escala.

Los drones o equipos UAV van equipados con cámaras de menor coste que las cámaras de fotogrametría utilizadas hasta ahora. Las innovaciones y evolución de la calidad de las cámaras digitales de pequeño formato y de las memorias de almacenamiento, hace que cada vez sea posible obtener mejores resultados de imagen en la aplicación de estos equipos.

El equipo utilizado en este proyecto es el denominado UX-5 de Trimble (Fig.7), que dispone de una cámara Samsumg NX-1000 de 21,6 Megapíxeles con la que se trabaja en enfoque al infinito para conseguir nitidez en las fotografías. El equipo UX-5 tiene una autonomía de vuelo de unos $60 \mathrm{~km}$, y es capaz de alcanzar una velocidad de crucero de $80 \mathrm{~km} / \mathrm{h}$. Además puede volar hasta con un viento de $65 \mathrm{~km} / \mathrm{h}$ y con lluvia ligera sin llegar a comprometer el trabajo. El sistema puede configurarse en tan solo 5 minutos.

El objetivo del proyecto fue la obtención de una ortofotografía del yacimiento, es decir, una imagen en la que no hay deformaciones ni efectos de perspectiva, sobre la que es posible realizar análisis métricos u obtención de documentos cartográficos. 


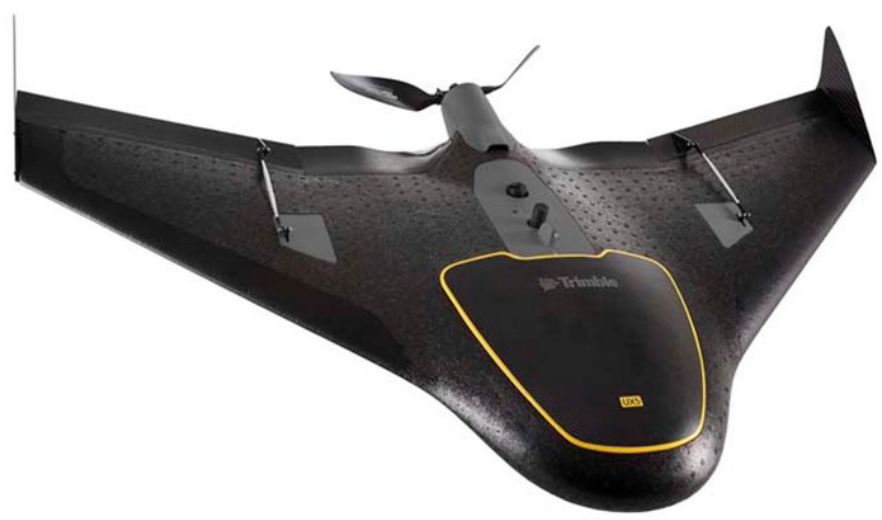

Fig.3. UX-5 Trimble.

Antes de llevar a cabo la toma de datos y la captura de imágenes, fue necesario realizar un proyecto de vuelo, tarea en la que se definieron los parámetros de configuración: altura de vuelo, recorrido que se realizará en el aire diseñando cada pasada, etc. A la hora de determinar estas variables, fue necesario tener en cuenta la calidad de la cámara fotográfica y la capacidad de vuelo del equipo UAV.

En este proyecto se programó un vuelo a $100 \mathrm{~m}$ de altura, contando con una autonomía de vuelo del equipo UAV de 55 minutos. Sobre el yacimiento se situaron unas dianas homogéneamente distribuidas, cubriendo toda la zona que se iba a sobrevolar. Se colocaron un total de 10 dianas distribuidas homogéneamente sobre toda la zona a sobrevolar. A estas dianas hubo que dotarlas de coordenadas geográficas en el sistema de coordenadas implantado anteriormente en el yacimiento. La tecnología utilizada para este trabajo fue la tecnología GNSS de posicionamiento global por satélite, utilizando la constelación GPS.

El equipo UX-5 de Trimble despega mediante un lanzador de unos $3 \mathrm{~m}$ de largo con un sistema similar a un tirachinas. Con la tableta de control del equipo UAV, en campo se controla la trayectoria de vuelo y el tiempo restante para que el dron aterrice. La zona de aterrizaje se selecciona antes del vuelo, y se programan los desplazamientos oportunos. Una vez obtenidas las fotografías, el trabajo en campo queda finalizado.

Se tomaron un total de 925 fotografías en 23 pasadas. En gabinete se orientan las fotografías con las imágenes de las dianas, y con sus coordenadas y se crean los modelos que representan el terreno a partir de la unión de todas ellas - Al igual que cuando se aplica fotogrametría convencional, estas imágenes se solapan unas a otras consiguiendo que todos los puntos del yacimiento aparezcan en al menos dos fotogramas. El solapamiento es necesario para conseguir la información en 3D de la zona, como sucede en cualquier sistema estereoscópico. 


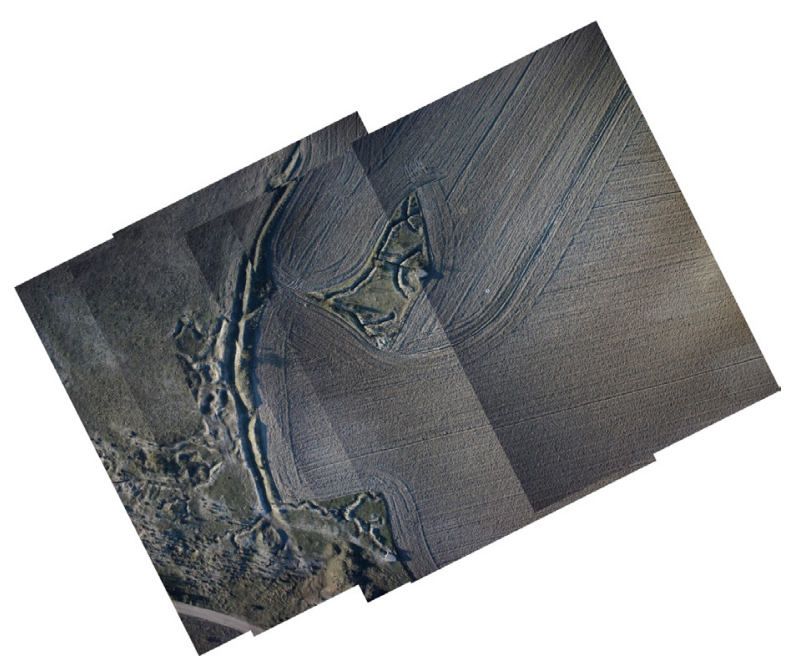

Fig.4. Solapamiento de fotografías para conseguir información 3D.

Mediante software específico, y puntos de control adicionales entre fotogramas, conseguiremos un modelo digital en tres dimensiones de la zona. Este modelo en 3D puede servir para fines múltiples, y es muy útil a la hora de mostrar el yacimiento, comprender la topografía del lugar y realizar análisis espaciales sobre él. Con las nuevas impresoras de 3D podemos obtener maquetas del área del levantamiento a la escala deseada.

Con este proyecto en particular se pretendió obtener una ortoimagen del yacimiento recurriendo a software de fotogrametría, para conseguir una imagen en la que podemos ver todos los detalles de la zona en vista cenital y sin deformaciones ni efectos de perspectiva, con un tamaño de pixel de 3,2 cm sobre el terreno.

\section{Realización de un modelo tridimensional del fortín no 3, mediante equipo laser escáner terrestre}

Las nuevas tecnologías, entre las que se encuentran los sistemas láser escáner 3D, están facilitando el trabajo de campo que en ocasiones es arduo. El proceso para la obtención del modelo 3D se fundamenta en las ciencias fotogramétricas, aunando a ellas la potencia de captura de los modernos equipos de modelización láser escáner 3D.

Este tipo de herramientas, a partir de un volumen de datos inmenso que puede filtrarse según las necesidades del proyecto, por un lado mejoran los resultados finales, y por otro abaratan los costes totales con tiempos de trabajo en campo y de necesidades de personal que se ven ampliamente reducidos. No obstante estas ventajas, presentan el inconveniente de que se aumenta el trabajo de oficina 
teniendo que invertir muchas horas en la edición y procesamiento de los datos. La precisión interna de los modelos es muy alta, pudiendo situarse en el orden milimétrico o incluso superior.

Las tecnologías láser escáner 3D en este proyecto se han utilizado para obtener el modelo tridimensional de un fortín de la Guerra Civil Española, el fortín pertenece al yacimiento arqueológico "Los Yesares", que como ya hemos mencionado se encuentra en Pinto ( Madrid), en el extremo noroeste del término municipal, próxima al Cerro de los Ángeles. La estructura está situada en un campo arado, orientado hacia el este. Está fabricado de hormigón y es de planta rectangular $(3,5 \times 2 \times 1,8 \mathrm{~m})$ con el frente redondeado y con una tronera que lo cubre. Presenta una inscripción de los constructores "12 $2^{a}$ compañía de Ingenieros 1aㅗ sección". Su acceso al interior es a través de una trinchera.

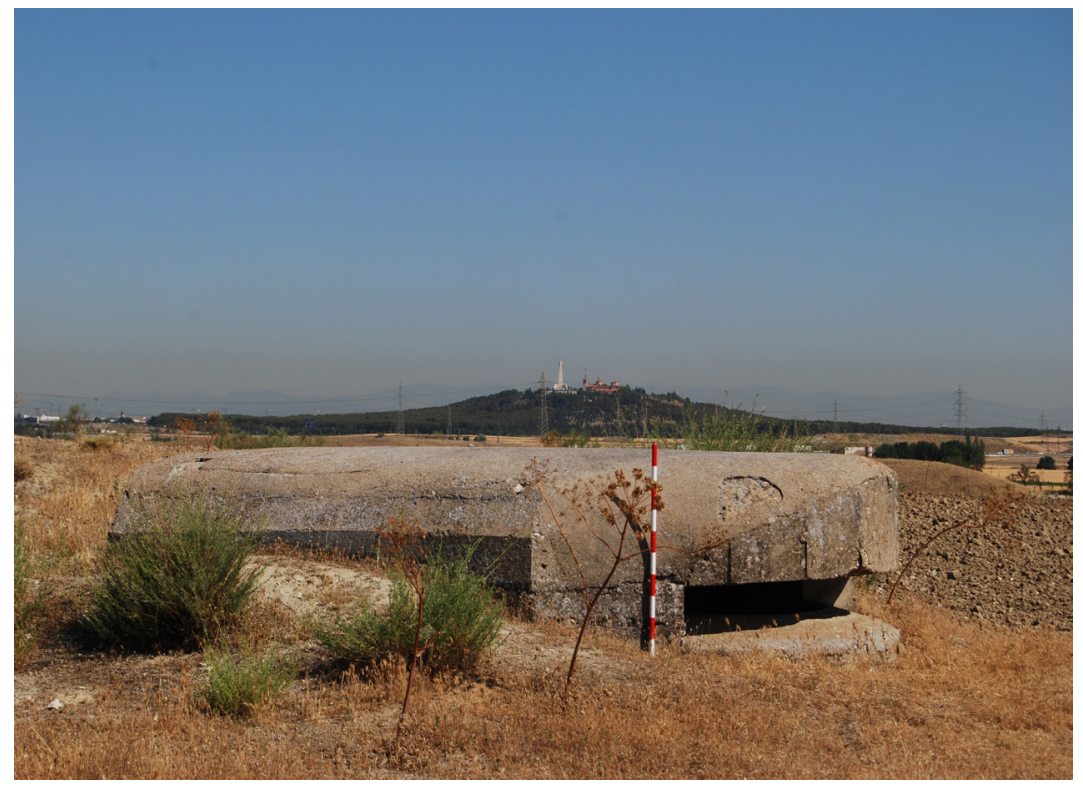

Fig.5. Fortín 3 de Los Yesares.

Para la realización del levantamiento 3D se ha utilizado el equipo láser-escáner IMAGER 5010. Antes de empezar el proceso de escaneado, se supervisó la zona del levantamiento (el fortín y sus alrededores) y se situaron dianas en ella, eligiendo las estaciones en las que posteriormente iba a ubicarse el equipo láser escáner, de manera que todo la estructura quedase cubierta y de modo que cada escaneo tuviese una zona común o recubrimiento (un mínimo de tres dianas) con los adyacentes, para después poder llevar a cabo la fusión de las nubes de puntos a un único sistema de representación. En este proyecto, para cubrir toda la zona de interés fueron necesarios 8 escaneos. Cada uno de ellos tuvo una duración de 
captura de unos 6 minutos, dependiendo el tiempo de captura de la resolución que requiera el modelo.

Al terminar cada escaneo, se puede visualizar en la pantalla digital del equipo láser escáner, la nube de puntos que se ha capturado, pudiendo comprobarse la idoneidad de los resultados en relación las necesidades del trabajo.

En campo es necesario llevar a cabo trabajos complementarios de topografía clásica, para poder optar a obtener el trabajo georreferenciado. Para ello se estacionó en tres vértices del yacimiento utilizando estaciones totales topográficas, de manera que desde estos puntos se observasen todas las dianas que se habían situado en la zona del levantamiento. Desde cada estación se observaron tres vértices que perteneciesen a la red local del yacimiento, pudiéndose calcular así mediante el método de intersección múltiple las coordenadas de las estaciones y a través de éstas, las coordenadas de todas las dianas en el sistema de referencia del proyecto. Para dotar de coordenadas a estos puntos, la red local se había enlazado previamente con la red REGENTE, marco geodésico del sistema geodésico nacional.

El trabajo de cálculo y edición comienza con la fusión de las nubes de puntos (Fig.6). Esta fase se ha realizado desde el programa propio del equipo láser-escáner. En él se crea un proyecto en el que se importan los escaneos, y en cada uno de los escaneados han de identificarse las dianas que han sido registradas en la observación, incluyendo sus correspondientes números de identificación. A continuación se fusionan todos los escaneos, con la opción "register project with targets", y se obtiene un informe en el que se indican las precisiones del registro de las dianas . En esta etapa de procesamiento de los datos es necesario contar con croquis y anotaciones realizadas en campo. Especialmente es necesario prestar atención a la numeración e identificación de las dianas ya que es muy fácil confundirlas entre sí. Al hacer la fusión de las nubes de puntos de los diferentes escaneados, es posible introducir en el modelo (como archivo de texto) las coordenadas de cada una de las dianas, quedando así el modelo final fusionado y además georreferenciado. En todo este proceso es necesario analizar las desviaciones típicas resultantes de los ajustes para el cálculo de cada una de las dianas, si alguna desviación excede la tolerancia establecida para el trabajo, se eliminará la diana correspondiente y se repetirá el cálculo completo de manera iterativa hasta obtener unos resultados válidos.

Una vez finalizada la georreferenciación y la fusión de los modelos, comienza el proceso de edición, propiamente dicho. El equipo láser-escáner utilizado puede captura un millón de puntos por segundo, con la resolución de captura "super hight" utilizada, la duración de escaneo e de 6 minutos, por tanto cada escaneo llegará a tener un peso aproximado de 1GB. Para poder manejarlo en las siguientes fases de edición, será necesario filtrar el modelo resultante antes de exportarlo al programa que vaya a utilizarse. Estos filtros, permiten realizar 
las tareas de limpieza del modelo, eliminando puntos innecesarios tales como los que corresponden al cielo o a elementos u obstáculos del momento de toma de datos, puntos que estén excesivamente próximos entre sí, etc. Tras el proceso de fusión y limpieza inicial, se exportan los datos al programa de edición. La finalidad del uso de este programa será completar la limpieza de toda la zona del modelo de árboles, arbustos, personas, y llevar a cabo la triangulación y la texturización del modelo.

Como producto final se han obtenido nubes de puntos, superficies trianguladas, ortoimágenes e incluso llegamos a obtener animaciones, productos 2D y 3D, que pueden ser muy interesantes desde el punto de vista arqueológico. Además todo estará georreferenciado y escalado, pudiendo hacerse medidas de distancias en cualquier parte del modelo 3D y otro tipo de análisis métricos.

\section{Bibliografía}

J.M. ARÉVALO: "La fortificación de campaña en la Guerra Civil Española". Revista de Historia Militar, 98, (2005).

F.J. LÓPEZ FRAILE: "La infografía 3D como sistema de documentación y divulgación". Técnicas aplicadas a la Arqueología. Estudios de diseño gráfico, (2007).

M. FARJAS ABADÍA: "Las Ciencias cartográficas en la arqueología: La búsqueda de la métrica en los modelos de divulgación científica". DATUM XXI, 3 (2003), 4-12.

M. FARJAS ABADÍA y F.J. GARCÍA-LÁZARO: Modelización Tridimensional y Sistemas Laser Escáner aplicados al Patrimonio Histórico. La Ergástula, Madrid: 2008. M. FARJAS ABADÍA: La topografía y sus métodos: Principios de investigación. Astrolabio, Bilbao: 2012.

M. FARJAS, D. BELLIDO y L. GONZÁLEZ: Tecnología UAV en yacimientos arqueológicos. Análisis de precisión de los modelos digitales de superficie y de los productos fotogramétricos derivados. Publicia, Saarbücken (Alemania): 2014. 